\title{
Volemic Resuscitation in a Patient with Multiple Traumas and Haemorrhagic Shock. Anti-oxidative Therapy Management in Critical Patients. A Case Report
}

\author{
Bedreag Ovidiu Horea ${ }^{1,2}$, Rogobete Alexandru Florin ${ }^{1,2^{*}}$, Sarandan Mirela ${ }^{3}$, Cradigati Alina Carmen ${ }^{3}$ \\ Nartita Radu1, Sandesc Dorel ${ }^{1,2}$, Papurica Marius ${ }^{1,2}$ \\ Clinic of Anaesthesia and Intensive Care, Emergency County Hospital "Pius Brinzeu", Timisoara, Romania \\ 2 Faculty of Medicine, "Victor Babes" University of Medicine and Pharmacy, Timisoara, Romania \\ ${ }^{3}$ Clinic of Anaesthesia and Intensive Care "Casa Austria", Emergency County Hospital "Pius Brinzeu", Timisoara, Romania
}

A patient with multiple traumas is usually found in severe haemorrhagic shock. In $40 \%$ of the cases, the patient with multiple traumas and haemorrhagic shock cannot recover due to secondary injuries and complications associated with the shock. In this paper we present the case of a male patient 30 years old, who suffered a car accident. The patient is admitted in our hospital with haemorrhagic shock due to femur fracture, acute cranial-cerebral trauma and severe thoracic trauma with bleeding scalp wound, associated with lethal triad of trauma. The clinical and biological parameters demand massive transfusion with packed red blood cells (PRBCs), fresh frozen plasma (FFP), cryoprecipitate $(\mathrm{CRY})$ and colloidal solution (CO) sustained with vassopresor for the haemodynamic stabilisation. During his stay in the ICU, the patient benefits from anti-oxidative therapy with Vitamin C, Vitamin E and Vitamin B1. After 14 days the clinical state of the patient improves and he is transferred in Polytrauma Department.

Keyword: fluids therapy, anti-oxidative therapy, oxidative stress, free radicals, shock index

Received: 30 October 2015 / Accepted: 05 January 2016

\section{Introduction}

The hypovolemic shock is present in most cases of severe polytrauma. In $40 \%$ of the cases, the patient with multiple traumas and haemorrhagic shock cannot recover due to secondary injuries and complications associated with the shock $[1,2]$. It has been proven that massive infusion of crystalloid solution is directly worsening the clinical picture of the patient through increased length of stay in the ICU, hyper- inflammation, infections complications, multiple organ failure and in the end death. The systemic oxidative stress that results is due to a series of factors: severe infections, tissue ischemia, prolonged hypoxia, hypovolemia, massive infusion of crystalloid solutions, acidosis, hypothermia; it represents a determinant factor of multiple organ dysfunctions [3-5]. In a critical patient, the level of antioxidants is low, needing exogenous supplementation for rebalancing of the oxidant/antioxidant equilibrium [6].

\section{Case History}

We present the case of I.P.V., male patient, 30 years old, $180 \mathrm{~cm}$ tall, $80 \mathrm{~kg}$, blood type $\mathrm{O}$ (I) Rh positive, admitted in the Emergency Room (ER) of the Emergency County Hospital Timisoara, following a car accident. The Injury Severity Score (ISS) was 38.

\section{Emergency Room (ER)}

In the ER investigations are proceeded according to the protocols for patients with multiple traumas. Clinically, the patient presents: heart rate (HR) 142 beats/min, arterial pressure (AP) $75 / 53 \mathrm{mmHg}$, respiratory rate 18 breaths/min, blood oxygen level $\left(\mathrm{SpO}_{2}\right) 92 \%$, central temperature 34.1 ${ }^{\circ} \mathrm{C}$. The biological tests show: haemoglobin (HB) 4,1 mg/ $\mathrm{dL}$, haematocrit $12 \%$, thrombocytes $78 \times 10^{3} \mathrm{UL}$, creatine kinase (CK) $5456 \mathrm{U} / \mathrm{L}$ and $(\mathrm{CKMB}) 79 \mathrm{U} / \mathrm{L}$, lactate dehydrogenase (LDH) $1392 \mathrm{U} / \mathrm{L}$, prolongation of the prothrombin time (PT) 15,9 s, international normalized ratio (INR) 2.5, partial thromboplastin time (PTT) 64\%, arterial blood gases (ABG) parameters: $\mathrm{pH} 7.12, \mathrm{paCO}_{2} 51 \mathrm{mmHg}, \mathrm{paO}_{2}$ $87 \mathrm{mmHg}, \mathrm{HCO}_{3}-20.1 \mathrm{mmol} / \mathrm{L}$, bases in excess $\mathrm{BE}(\mathrm{B})-$ $16,1 \mathrm{mmol} / \mathrm{L}, \mathrm{Na}^{+} 132 \mathrm{mmol} / \mathrm{L}, \mathrm{K}^{+} 3.4 \mathrm{mmol} / \mathrm{L}$, glucose (GLU) $184 \mathrm{mg} / \mathrm{dL}$, lactate (LAC) $5.9 \mathrm{mmol} / \mathrm{L}$.

The imagistic and radiologic exam show acute craniocerebral traumatism: bleeding scalp wound, right sphenoid hemosinus, cerebral hemorrhage, frontal contusion, contusion of the corpus callosum, diffuse axonal injury; severe thoracic traumatism: multiple rib fractures (II-VII), bilateral hemothorax, bilateral pulmonary contusions, tracheobronchial aspiration syndrome, stern fracture in third medium side; left femur fracture.

In ER the patient is orotracheally intubated and bilateral pleural drainage is performed. The primary volemic resuscitation is initialized through the administration of $3500 \mathrm{~mL}$ crystalloid fluid (CF) and $400 \mathrm{~mL}$ colloidal fluid (CO) (Figure 1). 


\section{Operating Room (OR)}

After approximately 2 hours from the moment of trauma, the patient is transferred in the OR for surgical treatment of the lesions. When admitted in the OR, the patient is in severe haemorrhagic shock (HR 144 beats/min, AP 65/40 $\mathrm{mmHg}, 5.2 \mathrm{mg} / \mathrm{dL} \mathrm{HB})$ and hypothermia $\left(34.2{ }^{\circ} \mathrm{C}\right)$. An antioxidative therapy is initiated through the administration of $1500 \mathrm{mg}$ of Vitamin $\mathrm{C}$ and volemic resuscitation through massive transfusion of $3200 \mathrm{~mL}$ PRBCs, $2000 \mathrm{~mL}$ fresh frozen plasma (FFP), $200 \mathrm{~mL}$ cryoprecipitate (CRY) and $500 \mathrm{~mL} \mathrm{CO}$ (Figure 1). Arterial blood pressure is sustained during the surgical intervention with vasopressor (Noradrenalin $4.5 \mathrm{mg} / \mathrm{h}$ ). The surgical therapy consists in the external fixation of the femur and hemostasis of scalp wound.

\section{Intensive Care Unit (ICU)}

The patient is admitted in the ICU after approximately 4 hours from the moment of the trauma. Clinically, the patient presents HR 112 beats/min, AP 112/69 mmHg, respiratory rate $16 \mathrm{breath} / \mathrm{min}, \mathrm{SpO}_{2} 100 \%$, central temperature $35,9^{\circ} \mathrm{C}$. Biologically it can be seen: anaemia (11.6 $\mathrm{mg} / \mathrm{dL} \mathrm{HB})$, thrombocytopenia (80x103 UL), lactic acidosis ( $4.4 \mathrm{mmol} / \mathrm{L} \mathrm{LAC)}$, rhabdomyolysis syndrome $(5598$ U/L CK, 81 U/L CKMB, 1432 U/L LDH), coagulopathy (14.5 s PT, 1.23 INR, $74 \%$ PTT). Volemic therapy with PRBCs, FFP and CRY infusion is continued (Figure 1).In the ICU the patient is mechanically ventilated following the corresponding parameters and he is clinically monitored continuously: invasive AP (systolic blood pressure SBP, diastolic blood pressure DBP, mean arterial pressure MAP), HR, temperature, ABG parameters and biological tests (Table I). In day 6 , the patient is tracheostomized and mechanical ventilation is continued according to the critical patient protocol. In day 9 the weaning is initiated, and after 2 days the patient is extubated. After 14 days in ICU, the patient is transferred in Polytrauma Department with normal clinical and biological parameters.

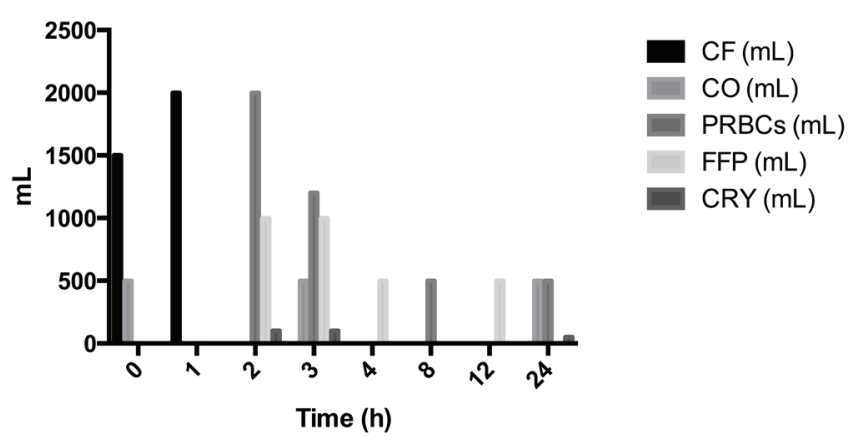

Fig.1. Fluid therapy management in the first $24 \mathrm{~h}$. Our infusion ratio was PRBCs:FFP:CRY = 1.4:1:0.08. ER = Emergency Room, OR = Operating Room, ICU = Intensive Care Unit, CF = crystalloid fluids, $\mathrm{CO}=$ colloidal fluids, $\mathrm{PRBCs}=$ packed red blood cells, FFP $=$ fresh frozen plasma, $\mathrm{CRY}=$ cryoprecipitate

\section{Discussion}

Shock Index (SI) and Modified Shock Index (MSI) are parameters used for appreciating the gravity of the haemorrhagic shock and moreover for a more precise management of the volemic resuscitation in the first 24 hours after the trauma. Expert studies prove that patients with SI $>1$ present a high rate of mortality. In addition, MSI $>1.3$ or MSI $<0.7$ are correlated with a higher rate of mortality as well. SI has been calculated with $\mathrm{SI}=\mathrm{HR} /$ $\mathrm{SBP}$, and $\mathrm{MSI}=\mathrm{HR} / \mathrm{MAP}$, where $\mathrm{MAP}=(2 \mathrm{DBP}+\mathrm{SBP}) / 3$ [7]. Volemic resuscitation with $\mathrm{CF}$ does not improve the IS and MIS in the first $2 \mathrm{~h}$. At the admission in OR, IS (2.2) and MIS (3) imposed a volemic resuscitation with blood and blood products without administering any crystalloid solutions. Moreover, diuretic drug was administered in order to remove excess of crystalloid. In ICU, after approximately 4 hours of the trauma volemic resuscitation was continued with blood compounds transfusions and CO. After 24 hours from the moment of the trauma, the patient is hemodynamic stable, presenting IS 0.7 and MSI 1 (Figure 2). A high percentage of the patients in the ICU suffer from sepsis. The severe sepsis accelerates biosynthesis of free reactive species (free radicals) in the tissue. At a critical patient, the equilibrium between the oxidants and antioxidants is disturbed, resulting in oxidative stress. The prolonged action of free radicals on mitochondria decrease

Table I. Evolution of biochemical parameters

\begin{tabular}{|c|c|c|c|c|c|c|}
\hline & $\begin{array}{c}\mathrm{HB}(\mathrm{mg} / \\
\mathrm{dL})\end{array}$ & INR & PT (s) & $\begin{array}{c}\text { APTT } \\
\%\end{array}$ & $\mathrm{pH}$ & $\begin{array}{c}\mathrm{LAC} \\
(\mathrm{mmol} / \mathrm{L})\end{array}$ \\
\hline O h (ER) & 4,1 & 2,5 & 15,9 & 64 & 7,12 & 5,9 \\
\hline $1 \mathrm{~h}(\mathrm{ER})$ & 5 & 2,6 & 15,1 & 63 & 7,11 & 6 \\
\hline $2 \mathrm{~h}(\mathrm{OR})$ & 5,2 & 2,3 & 16 & 62 & 7,13 & 5,8 \\
\hline $3 \mathrm{~h}(\mathrm{OR})$ & 7,8 & 2,2 & 15 & 63 & 7,21 & 5,2 \\
\hline $4 \mathrm{~h}$ (ICU) & 9,9 & 1,23 & 14,5 & 75 & 7,25 & 4,4 \\
\hline 8 h (ICU) & 11,6 & 1,18 & 14,2 & 77 & 7,28 & 3,1 \\
\hline $12 \mathrm{~h}(\mathrm{ICU})$ & 12,1 & 1,12 & 13 & 79 & 7,38 & 1,4 \\
\hline 24 h (ICU) & 12 & 1,11 & 12,7 & 81 & 7,41 & 0,98 \\
\hline
\end{tabular}

HB (hemoglobin); INR (international normalized ratio); PT (prothrombin time); APPT (activated partial thromboplastin time); LAC (lactic acid).

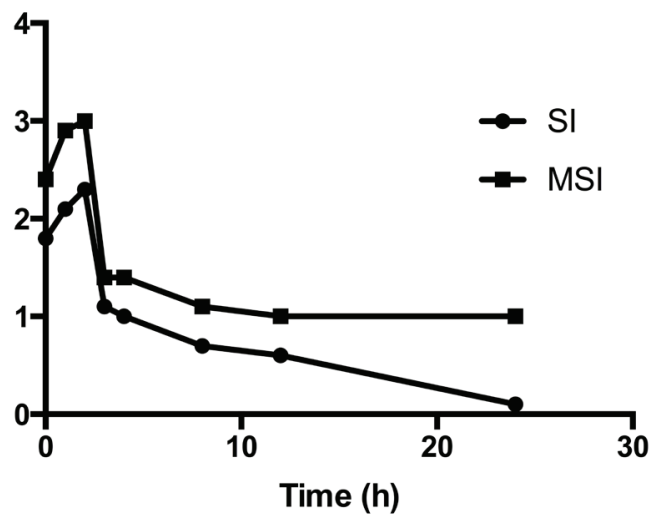

Fig. 2. The evolution in the first 24 hours after the trauma of Shock Index (SI) and Modified Shock index (MSI). It can be observed that once the massive infusions are initiated, the SI and MSI dramatically decreases, reaching the normal values after approximately 12 hours from the moment of the trauma 
energy production, promotes cellular apopthosis, tissue damage, organ failure and finally, death. Lorente et al, proves that a low level of total antioxidant capacity (TAC) is directly proportional with the risk of mortality in critical patients with sepsis [8]. Moreover, in patients with severe haemorrhagic shock, the production of free radicals is accelerated by ischemia - reperfusion syndrome. In our case, the antioxidative therapy consisted in the administration of: intravenous Vitamin C (1500 mg, 7 days), intravenous Vitamin E (600 mg in first day 1, $300 \mathrm{mg}$ in day 2 to 5 ) and intravenous Vitamin B1 (305 mg in first day, $205 \mathrm{mg}$ in day 2 to 5 ).

Fluid therapy management in a critical patient with severe haemorrhagic shock imposes a series of parameters that need to be monitored: lactate level, urine output, HR, SI and MSI. Good management of fluid resuscitation implies decrease of lactate level, HR, SI, MSI, lower doses of vasopressor and increase of urine output and MAP. In our case, the aggressive resuscitation in the OR (massive blood and blood products transfusion), diuretic therapy and antioxidant therapy ensured the success of recovery, despite an unappropriated volemic resuscitation on the ambulance and in the ER (first 2h). Delayed administration of blood and blood products and $\mathrm{CO}$, requires administration of vasopressors to maintain MAP in OR.
In conclusion, we can affirm with certainty that inadequate and/or delayed fluid therapy can significantly reduce surviving in haemorrhagic shock.

\section{Conflict of interest}

Nothing to declare

\section{References}

1. Malbrain ML, Marik PE, Witters I, et al. Fluid overload, de-resuscitation, and outcomes in critically ill or injured patients: a systematic review with suggestions for clinical practice. Anaesthesiol Intensive Ther. 2014;46:361-80.

2. Curry N, Davis PW. What's new in resuscitation strategies for the patient with multiple trauma? Injury. 2012;43:1021-8.

3. Kozlov AV, Bahrami S, Calzia E, et al. Mitochondrial dysfunction and biogenesis: do ICU patients die from mitochondrial failure? Ann Intensive Care. 2011;1:41.

4. Edwards MR, Mythen MG. Fluid therapy in critical illness. Extrem Physiol Med. 2014;3:16.

5. Barbosa Neto JO, Breda de Moraes MF, Souza Nani R, Rocha Filho JA, Carvalho Carmona MJ. Hemostatic resuscitation in traumatic hemorrhagic shock: case report. Rev Bras Anestesiol. 2013;63:99-102.

6. Giladi AM, Dossett L, Fleming SB, Abumrad NN, Cotton B. High-dose antioxidant administration is associated with a reduction in post-injury complications in critically ill trauma patients. Injury. 2011;42:78-82.

7. Singh A, Ali S, Agarwal A, Srivastava RN. Correlation of shock index and modified shock index with the outcome of adult trauma patients: a prospective study of 9860 patients. N Am J Med Sci. 2014;6:450-2.

8. Lorente L, Martín MM, Almeida T, et al. Association between serum total antioxidant capacity and mortality in severe septic patients. J Crit Care. 2015;30:217.e7-12. 